Rapport - Société canadienne d'histoire de l'Église catholique

\title{
Parlementaires de la région et leur rôle dans l'Église
}

\section{Lionel Bertrand}

Volume 31, 1964

URI : https://id.erudit.org/iderudit/1007347ar

DOI : https://doi.org/10.7202/1007347ar

Aller au sommaire du numéro

Éditeur(s)

La Société canadienne d'histoire de l'Église catholique

ISSN

0318-6148 (imprimé)

1927-7075 (numérique)

Découvrir la revue

Citer cet article

Bertrand, L. (1964). Parlementaires de la région et leur rôle dans l'Église. Rapport - Société canadienne d'histoire de l'Église catholique, 31, 65-72.

https://doi.org/10.7202/1007347ar

Tous droits réservés @ La Société canadienne d'histoire de l'Église catholique, 1965
Ce document est protégé par la loi sur le droit d'auteur. L’utilisation des services d'Érudit (y compris la reproduction) est assujettie à sa politique d'utilisation que vous pouvez consulter en ligne.

https://apropos.erudit.org/fr/usagers/politique-dutilisation/ 


\title{
Parlementaires de la région et leur rôle dons l'Église
}

\author{
L'honorable Lionel Bertrand rappelle dans sa causerie le \\ souvenir d'hommes d'Etat de sa région qui ont bien servi \\ l'Eglise et la Société, dont Sir Adolphe Chapleau, l'honorable \\ Athanase David, l'honorable Hector Perrier, l'honorable Paul \\ Sauvé, etc. \\ Notons que Sir Adolphe Chapleau et l'honorable Paul \\ Sauvé furent tous deux premiers ministres de la Province de \\ Québec.
}

"Parlementaires de la région, et leur rôle dans l'Eglise ": voici le titre d'une causerie qui fera revivre devant vous quelques figures d'hommes publics qui, au cours du dernier et même au cours du présent siècle, ont dans ce secteur du Québec où nous sommes, largement mérité de l'Etat, mais mérité aussi de l'Eglise dont ils furent à des époques diversifiées, de constants appuis, de constants défenseurs.

L'histoire politique du comté de Terrebonne, qui remonte à la Chambre d'Assemblée de 1792 et dont les limites sont demeurées les mêmes depuis la Confédération, est, certes, l'une des plus riches et des plus palpitantes. De 1830 à 1838, le comté de Terrebonne fut représenté par Sir Louis-Hippolyte Lafontaine, dont il serait superflu de donner la biographie. Sous l'Union, soit de 1841 à 1867 , on retrouve encore Sir Louis-Hippolyte Lafontaine, pour quatre années de 1844 à 1848, et, par la suite, des députés qui ont laissé leurs traces dans l'histoire, l'honorable Louis-Michel Viger, l'honorable Augustin NorbertMorin, l'honorable Louis-Siméon Morin, Louis Labrèche-Viger, pour ne nommer que ceux-ci.

En 1867, le comté de Terrebonne fournissait à la politique provinciale un parlementaire excessivement puissant : Sir Adolphe Chapleau, dont je voudrais dès maintenant résumer l'étincelante carrière. Il naquit à Sainte-Thérèse le 9 novembre 1840. Admis au barreau le 2 décembre 1861, à l'âge de 21 ans, il entrait en politique en 1867, et par acclamation devenait député conservateur de Terrebonne, au parlement de Québec. Il avait alors 27 ans. Six ans plus tard, en 1873, à l'âge de 33 ans, il entre dans le cabinet Ouimet comme Solliciteur général, et en 1876 devenait Secrétaire de la Province dans le cabinet de Boucherville.

En 1878, à l'âge de 38 ans, il était devenu le chef du parti conservateur, et de 1879 à 1882 , il sera le premier ministre de la province de Québec.

En 1882, à l'âge de 42 ans, il quitte l'arène provinciale et se porte candidat dans Terrebonne, mais cette fois sur le plan fédéral. Il est 
Secrétaire d'Etat dans le gouvernement de Sir John Macdonald, puis ministre des Douanes, sous Sir John Abbott.

\section{Québec.}

De 1892 à 1898 , il est le Lieutenant-gouverneur de la province de

Il fut vingt-cinq années député de Terrebonne, quinze ans au provincial et dix ans au fédéral. Pendant ce quart de siècle, Chapleau fut de toutes les luttes fédérales et provinciales, et jamais orateur n'attira autant de foules aux assemblées où il apparaissait. Son éloquence était proverbiale, et on venait de ìoin expressèment pour İentendre.

En dehors des campagnes électorales il a prononcé des discours qui sont des chefs-d'œuvre sur le plan humain, religieux et patriotique. A preuve, cette mémorable allocution qu'il prononçait à l'inauguration du nouveau Séminaire de Sainte-Thérèse, le 26 juin 1883, et dans laquelle il disait avec combien d'émotion son admiration pour le clergé. En voici quelques extraits :

« Je suis dans cette position difficile de celui qui ne s'attend à aucun merci : j'ai trop souvent été appelé à prendre la parole pour réclamer efficacement votre indulgence. Cependant ce n'est pas impunément que l'on parle devant une assemblée aussi brillante, sans préparation, et je n'en ai pas. Mais j'ai cette préparation du cœur qui se souvient. Je sais ce que la société doit à la religion qui la dirige, la protège et la soutient.

«Enfant de cette paroisse, né dans ce comté, depuis dix-sept ans travaillant aux affaires du gouvernement de mon pays, j'ai cru devoir venir ici témoigner aux membres de cette grande institution du Petit Séminaire de Sainte-Thérèse mon admiration pour eux et le plaisir que j'ai de voir cette maison naguère éprouvée par le feu mais non pas détruite. Non, le Séminaire de Sainte-Thérèse n'a pas été détruit, il a toujours vécu et on peut lui appliquer la belle phrase d'Horace : Monumentum are perennius : ceux qui en verront la ruine n'ont pas vu le jour. Le Séminaire de Sainte-Thérèse, c'est à la fois le dévouement, la sagesse et la science de ceux qui en sont encore aujourd'hui les fondations et les murs indestructibles.

"Je désirerais venir comme membre de l'Etat et de la société civile affirmer ici que la maison d'éducation est le terrain où l'Eglise et l'Etat doivent se rencontrer pour s'entendre. La société a pour premier devoir de nourrir et d'élever ses enfants, de conserver les traditions de la morale, de la science et du travail : elle a pour premier devoir d'instruire ses membres.

"Dans les sociétés chrétiennes, l'Etat a toujours laissé la haute main de l'éducation à l'Eglise. Dans les sociétés catholiques, on lui en a toujours laissé la direction, et les gouvernements ont eu raison.

« L'Etat travaille pour la prospérité matérielle du peuple en confiant à la religion le soin de diriger les consciences, et les consciences dirigées par la religion sont les plus fermes appuis de l'Etat. L'Etat n'a pas 
besoin de craindre ceux qui enseignent la belle parole du Christ : "Rendez à César ce qui appartient à César et à Dieu ce qui appartient à Dieu. " L'Etat ne sera jamais en danger lorsqu'il confiera l'éducation de la jeunesse à ceux qui disent et professent que tout pouvoir vient de Dieu. Il n'aura jamais besoin de craindre le pouvoir de l'Eglise, car ce pouvoir béni est celui que Dieu lui-même a indiqué aux gouvernements.

"Messieurs, je suis venu pour vous dire cela, et je le dis, parce que je le crois. Je ne souhaite qu'une seule chose pour mon pays, c'est, Messeigneurs et vous, vénérables membres du clergé, c'est qu'il lui soit donné de toujours suivre vos enseignements et mériter vos bénédictions. »

Des paroles aussi émouvantes, prononcées par des hommes d'Etat dont le prestige était indéniable, trouvent dans notre monde moderne, lorsqu'elles sont rappelées, un écho bienfaisant. A cette époque, il y a quatre-vingt-deux ans, en dépit de luttes politiques excessivement violentes et passionnées, les chefs d'Etat saluaient le clergé avec admiration, et concevaient qu'au-dessus des forces matérielles trop fréquemment chancelantes, il y avait celles qui sont les plus puissantes de toutes, les forces spirituelles.

Mais Chapleau voyait plus grand, comme catholique. Il suffit de rappeler le fameux discours qu'il prononçait sur la Papauté, en février 1868 - il était député depuis un an et il avait 28 ans - à l'occasion du départ des zouaves pontificaux pour Rome. Déjà il affirmait cette foi qui ne devait jamais vaciller et que toute sa vie il devait avec fierté ne cesser d'affirmer :

"La vérité, pour nous catholiques, repose dans l'infaillibilité du pape et son infaillibilité est rigoureusement liée à son indépendance; son indépendance, dans les conditions actuelles de l'Europe, est liée à l'indépendance de Rome vis-à-vis toute autre souveraineté.

"C'est donc Rome qui sert aujourd'hui de base à cette colonne de la vérité qui s'élève jusqu'au ciel et sur laquelle Dieu s'appuie complaisamment pour répandre ses bienfaits sur la terre. C'est donc à Rome que doivent se rendre les soldats de la vérité, à Rome que nos jeunes compatriotes vont offrir leur courage, leurs vœux, leur sang, leur vie même !

"Ce mouvement tout spontané les honore et nous honore nousmêmes; mais en même temps il nous permet de montrer notre reconnaissance à la papauté, à cette institution qui a formé l'Europe, relevé l'ancien monde enfoui dans la poussière, animé la civilisation ancienne du souffle du christianisme, et grandi l'humanité de toute la hauteur de la vérité révélée.

«La vérité, c'est l'âme du monde. Du moment qu'elle disparaîtra de la terre, les temps seront accomplis et les nations seront dispersées. Incertaine, indécise pendant quatre mille ans, elle s'incarna un jour dans la personne du Christ, qui en confia la garde à son Vicaire. 
« La papauté qui a déjà sauvé l'Europe des envahissements de la barbarie, sauvera le monde de l'invasion plus redoutable des nouveaux barbares qui la menacent aujourd'hui. Le socialisme mine sourdement les trônes de l'Europe, et c'est un châtiment que peut-être la Providence réserve à la politique sceptique du siècle. Aux élans de la foi qui faisaient la beauté du sentiment public du moyen âge, on a substitué le froid calcul de la diplomatie, et on s'en est applaudi. Dieu qui voit et conduit tout d'en haut se venge de la raison humaine s'insurgeant contre ses volontés. On sent le monde crouler; les uns n'espèrent plus, les autres dédaignent, et se laissent aller dans l'indifférence. Mais pourquoi cette dissolution? C'est que les gouvernements n'ont plus de foi, c'est parce que l'on a répandu dans le corps social des éléments putrides qui, en produisant la fermentation, ont amené la putréfaction et par suite la dissolution. Le doute, le matérialisme ont voulu fouiller dans toutes ses parties l'être moral, social et politique; on a tout remué, tout déplacé, et quand on a voulu refaire, on s'est aperçu que les pièces disloquées ne s'ajustaient plus; elles avaient déjà subi l'action délétère du doute qui engendre le découragement, le désespoir, et comme dernier résultat les révolutions...

«Ne perdons pas cependant l'espérance; la foi vive des nations nouvelles de ce continent régénérera le monde, en se retrempant aux sources premières de la chrétienté. C'est un devoir pour nous de savoir apprécier ce que la papauté a fait pour la civilisation, un devoir que l'on ne peut mieux accomplir qu'en offrant pour sa défense le sang le plus pur de notre nation. Qu'ils partent donc, nos zouaves, et qu'ils comptent sur notre encouragement, nos sympathies et nos affections...

" J'ai parlé de civilisation. J'ai dit que les papes avaient le plus contribué à la développer en Europe. L'incrédulité va sourire de cette prétention; qu'elle se tranquilise. Car je n'entends parler nullement ici de notre civilisation à la vapeur et à l'électricité, ni des merveilles de l'industrie contemporaine; j'entends plutôt parler de la civilisation qui donne aux peuples un caractère moral et religieux, et qui produit lentement des œuvres que le temps ne détruira jamais."

Dans son discours-programme prononcé à Sainte-Thérèse, le 3 novembre 1881, à l'ouverture de la campagne électorale, et parlant alors comme chef de parti et comme premier ministre, Chapleau aura ces paroles qui méritent d'être citées :

"Messieurs, quant à la ligne de conduite que j'entends suivre dans l'avenir en matière religieuse, je dois vous dire que dans toutes les questions d'ordre purement religieux qui s'élèveront, je me conduirai conformément aux décisions de nos évêques. Ils ont la sagesse et l'expérience; et la hauteur de leur mission nous est une garantie de cette loyauté dont ils ont déjà donné tant d'exemples. En bons catholiques, nous devons nous soumettre à leurs décisions; en bons Canadiens, nous 
leur devons reconnaissance pour les immenses services que l'histoire du pays et la mémoire du peuple ont mis à leur crédit. 》

En 1881, Sa Sainteté le pape Léon XIII le créait Commandeur de l'Ordre de Saint-Grégoire-le-Grand.

Chapleau habita Sainte-Thérèse la majeure partie de sa vie dans une maison qui existe encore, rue Saint-Charles, aux abords de la ville; elle est occupée aujourd'hui par M. Claude Calvé qui, depuis janvier dernier, est concierge à l'école Adolphe-Chapleau, institution ainsi nommée par la Commission scolaire de la ville de Sainte-Thérèse pour rendre un hommage particulier à l'un des Térésiens les plus méritants.

Plus près de nous, je voudrais mentionner le sénateur Jules-Edouard Prévost, l'un des journalistes les plus brillants de son temps, fondateur du journal "L'Avenir du Nord» de Saint-Jérôme, et l'un des citoyens les plus illustres de notre région.

Il appartenait à cette famille des Lions du Nord. Né le 21 novembre 1871 , il fut très jeune en vedette dans cette famille, l'une des plus remarquables des pays d'en haut. Elu à la Chambre des Communes en 1917, il était rélu aux élections générales de 1921, 1925 et 1926. Nommé au Sénat le 3 juin 1930, il décédait en 1943, à l'âge de 72 ans.

S'il fut membre du Conseil de l'Instruction publique dès 1910, il n'en fut pas moins, pour de longues années, membre de la Commission scolaire de Saint-Jérôme, et dans le domaine de l'éducation, sur le double plan provincial et local, il rendit des services signalés. Nous n'avons malheureusement pas le texte d'aucun de ses discours, mais dans son journal, que d'articles apportant au clergé une solide coopération, et apportant à la cause de l'éducation un dévouement digne de tous les éloges.

Le sénateur Prévost était le frère cadet du R.P. Eugène Prévost, né le 24 août 1860, à Saint-Jérôme, élève du Séminaire de Sainte-Thérèse, fondateur de la Fraternité Sacerdotale et des Oblates de Béthanie, décédé en 1946, en odeur de sainteté, à l'âge de 86 ans.

Quand le père Prévost apprit, le 26 octobre 1943, la mort de son frère Jules-Edouard, il déclara :

« Je baise la croix, je m'y cramponne. C'est une partie de mon âme qui est partie pour l'éternité. Nous ne nous reverrons plus qu'en Jésus, comme nous nous sommes aimés en Lui sur la terre. Par le cœur, il faisait partie de la Congrégation. Il l'a aimée et servie fidèlement. »

En 1916, un jeune avocat devenait député provincial de Terrebonne, c'était Athanase David, fils du sénateur L.-O. David; nommé Secrétaire de la Province, département de qui relevait en premier lieu 
l'éducation et auquel devaient graduellement s'ajouter l'hygiène et l'assistance publique, il devait par la suite être élu député de Terrebonne et assumer les mêmes fonctions ministérielles en 1919, 1923, 1927, 1931 et 1935. Il ne fut pas candidat en 1936, mais faisait un retour triomphal en 1939, après trois ans d'absence de la scène politique, mais dès le 9 février 1940 il était nommé au Sénat.

Athanase David, pendant son long stage comme Secrétaire de la Province, donna à l'éducation son réel essor. Ses œuvres sont prodigieuses. En 1035 on pouvait dire de M. David: "Nous lui devons l'Assistance publique, le Service provincial d'Hygiène, la création de trente unités sanitaires, les Archives de la province, le Musée provincial, le placement familial, les écoles de rang, l'aide aux universités et aux collèges classiques, l'Ecole Technique, l'amélioration de l'enseignement primaire, etc."

Ce qui m'a toujours frappé chez M. David, et j'ai été dans son sillage pendant dix ans, c'était un semeur d'idées et un animateur de grandes réalisations. Il croyait à la nécessité de l'éducation et n'a cessé de la prôner; en une occasion il dira :

" Je dirai, convaincu que je reflète un sentiment que tous exprimeraient avec enthousiasme, que si l'enseignement d'un peuple est une condition de progrès et d'ordre dans tout pays, il est chez nous une condition sine qua non de survivance nationale. Il revêt à ce titre un tel caractère que négliger de le dispenser, même temporairement, ferait plus que nous affaiblir, ce serait nous tuer. C'est le moment de répéter que les peuples ne meurent pas mais se suicident. ”

"L'œuvre d'instruction, ajoutera-t-il, requiert l'union de toutes les volontés et de toutes les énergies et, ici surtout, la coopération de l'Eglise et de l'Etat s'impose, car aucune ouvre ne peut être féconde et durable sans avoir pour assises les deux forces qui animent notre vie nationale."

En 1936, Athanase David écrivait en parlant de l'avenir : «Ceux-là qui vivront à cette époque et qui liront dans l'Histoire nos inquiétudes d'hier, nos anxiétés d'aujourd'hui, répétant notre geste devant les ancêtres, s'inclineront devant ce que nous serons pour eux : des pionniers et des précurseurs."

Athanase David fut les deux à la fois. Réélu en 1939, il ne devait être député provincial que quelques semaines. Mais en 1940 lui succédait comme député de Terrebonne l'honorable Hector Perrier, associé professionnel de M. David, et M. Perrier devenait à son tour le Secrétaire de la Province. Il ne fut dans la politique active que quatre ans, mais nombreuses furent les initiatives prises dans le domaine scolaire, initiatives qui complétaient en certains points l'œuvre de M. David, et auxquelles s'ajoutaient avec audace des projets que les années se chargeront graduellement de matérialiser. Il était nommé juge de la Cour Supérieure en 1944. Monsieur Perrier avait été membre de la 
Commission des Ecoles catholiques de Montréal, membre du Conseil de l'Instruction publique de 1934 à 1940 , et il devait l'être par la suite de 1944 à 1964.

L'Union Nationale arrivait au pouvoir en 1944, et le successeur de M. Perrier au Secrétariat provincial fut l'honorable Omer Côté qui, lui aussi, fit ouvre féconde; nommé juge pour le district de Terrebonne, il choisissait comme lieu de domicile Sainte-Thérèse-de-Blainville.

En 1960, l'éducation trouvera, avec la venue du gouvernement Jean Lesage, un essor excessivement dynamique, et qui devait conduire, quatre ans plus tard, l'honorable Gérin-Lajoie, ministre de la Jeunesse en 1960 et chargé dès 1960 de toutes les matières relevant de l'éducation, à devenir le premier titulaire du nouveau ministère de l'Education.

Mais comment oublier ici le nom d'un parlementaire de la région, ancien élève de cette maison, l'honorable Paul Sauvé, député des DeuxMontagnes, ministre de la Jeunesse pendant plusieurs années. Il devenait au décès de $M$. Duplessis, le premier ministre de la Province, mais son règne fut de courte durée, de septembre 1959 à janvier 1960 .

Dans la nuit du $1^{\text {er }}$ janvier 1960 , il était brusquement emporté. Le matin, avec les membres de sa famille, il avait, dans la vieille et historique église de Saint-Eustache, reçu la communion des mains même de son curé $\mathrm{M}^{\mathrm{gr}}$ Louis-Joseph Rodrigue, l'un des conférenciers de ce congrès et ancien président de la Société Historique de Sainte-Thérèse. Homme d'Etat d'un grand prestige, au cœur généreux, Paul Sauvé toute sa vie aura été fidèle à sa foi, et l'aura prouvé en de multiples occasions. Nombreuses sont les allocutions où elle s'est traduite et nombreuses sont les initiatives qu'il a prises comme ministre de la Jeunesse envers l'éducation et envers le Séminaire de Sainte-Thérèse qu'il affectionnait particulièrement.

Certes, je ne veux mentionner que ces noms. Ils feront renaître de précieux souvenirs chez ceux des membres de notre Société qui ont un peu vieilli et qui sont à même aujourd'hui d'évaluer leurs æuvres. Pour ceux qui sont moins âgés, ils apprendront qu'à diverses époques de notre histoire politique, des parlementaires de taille n'ont pas craint de s'affirmer comme les collaborateurs de l'Eglise, et que fièrement ils l'affirmaient. Leurs actes publics n'ont jamais cessé de le prouver, et ils furent des modèles pour les populations qui les élisaient.

Il y a déjà vingt-trois ans que je siège dans les parlements. J'ai toujours soutenu que l'Eglise et l'Etat doivent marcher ensemble. L'Eglise demeure toujours l'outil indispensable pour maintenir la paix, pour donner à la vie son prix, son véritable but, pour établir la concorde, par la justice et la charité. Certes, nombreuses sont, dans ce siècle qui brûle les étapes, les idées qui jaillissent, qui troublent, qui 
empoisonnent parfois, mais on aura beau faire, on aura beau dire, les forces spirituelles demeureront indispensables. Je n'ai jamais cessé de défendre l'Eglise et d'appuyer ses œuvres, par la parole, mais aussi par le journal que je fondais il y a vingt-sept ans et que je dirige encore. En 1960, sur la recommandation de mon évêque, qui a toute ma coopération, le pape Jean XXIII, me remettait le bouton que je porte avec fierté à la boutonnière, celui de l'Ordre de Saint-Grégoire-leGrand. Comme Chapleau, à quatre-vingt-seize ans de distance, je crois que le Pape est encore, dans ce brouhaha du monde moderne, le phare dans la tempête. Le Pape demeure aujourd'hui comme hier, et peutêtre plus aujourd'hui qu'hier, la figure dominante d'un univers inquiet et tourmenté. Il domine les tempêtes que soulèvent l'ambition des grands et la cupidité des nations. Et les nations pourtant n'entendent jamais sa voix sans tressaillir. Mêlée de près ou de loin à tous les grands événements du monde, la papauté ne cesse de recommander au monde la civilisation qui est la meilleure de toutes puisqu'elle est basée sur la charité, la compréhension, le respect de la dignité humaine, et sur le commandement qui résume tous les autres: Aimez-vous les uns les autres!

Que notre Société vive et progresse, c'est le souhait que je formule. Elle tient son congrès annuel dans ce comté de Terrebonne qui est le mien, dans ma propre ville de Sainte-Thérèse, dans ce séminaire où j'ai fait mes études. De tout cela je me réjouis. Ecrire l'histoire de l'Eglise, butiner l'histoire de l'Eglise, y a-t-il quelque chose de plus palpitant? Aimer l'histoire de l'Eglise, c'est comprendre encore mieux le rôle que l'Eglise joue depuis dix-neuf siècles, et comprendre mieux aussi la lumineuse décision de Jean XXIII, reprise par Paul VI, de rajeunir l'Eglise, pour qu'elle continue sa mission, mission indispensable au monde jusqu'à la fin des temps.

Hon. Lionel Bertrand, C.O.St-G.G. Sainte-Thérèse-de-Blainville, P.Q. 